\title{
Spin-Crossover Phenomenon in a Pentanuclear Iron(II) Cluster Helicate
}

Zheng Yan, ${ }^{\dagger, \downarrow, l}$ Wei Liu, ${ }^{\dagger, \|}$ Yuan-Yuan Peng, ${ }^{\dagger}$ Yan-Cong Chen, ${ }^{\dagger}$ Quan-Wen Li, ${ }^{\dagger}$ Zhao-Ping $\mathrm{Ni}^{\dagger}{ }^{\dagger}$ Ming-Liang Tong ${ }^{\dagger} *$

${ }^{\dagger}$ Key Laboratory of Bioinorganic and Synthetic Chemistry of Ministry of Education, School of Chemistry and Chemical Engineering, Sun Yat-Sen University, Guangzhou 510275, P. R. China

${ }^{\ddagger}$ College of Biological, Chemical Sciences and Engineering, Jiaxing University, Jiaxing, Zhejiang 314001, P. R. China

\| These authors contributed equally to this work 


\section{Contents of the Supporting Information}

Figure S1. MS spectra of the $\left[\left\{\mathrm{Fe}^{\mathrm{II}}(\mu-\mathrm{bpt})_{3}\right\}_{2} \mathrm{Fe}_{3}{ }_{3}\left(\mu_{3}-\mathrm{O}\right)\right]^{2+}$ cation.

Figure S2. Thermogravimetric-mass spectroscopy analysis.

Figure S3. Packing diagram showing heterochiral layers in the structure.

Figure S4. Plots of $\chi_{\mathrm{M}} T$ vs $T$.

Table S1. The values of trigonality index $\tau$ for $\mathrm{Fe} 3 \sim \mathrm{Fe} 5$ and $\mathrm{Fe} 6$.

Table S2. Intermolecular interactions around two apical [ $\left.\mathrm{Fe}^{\mathrm{II}}(\mathrm{bpt})_{3}\right]^{-}$units at $293 \mathrm{~K}(150 \mathrm{~K})$.

Table S3. Mössbauer hyperfine parameters 


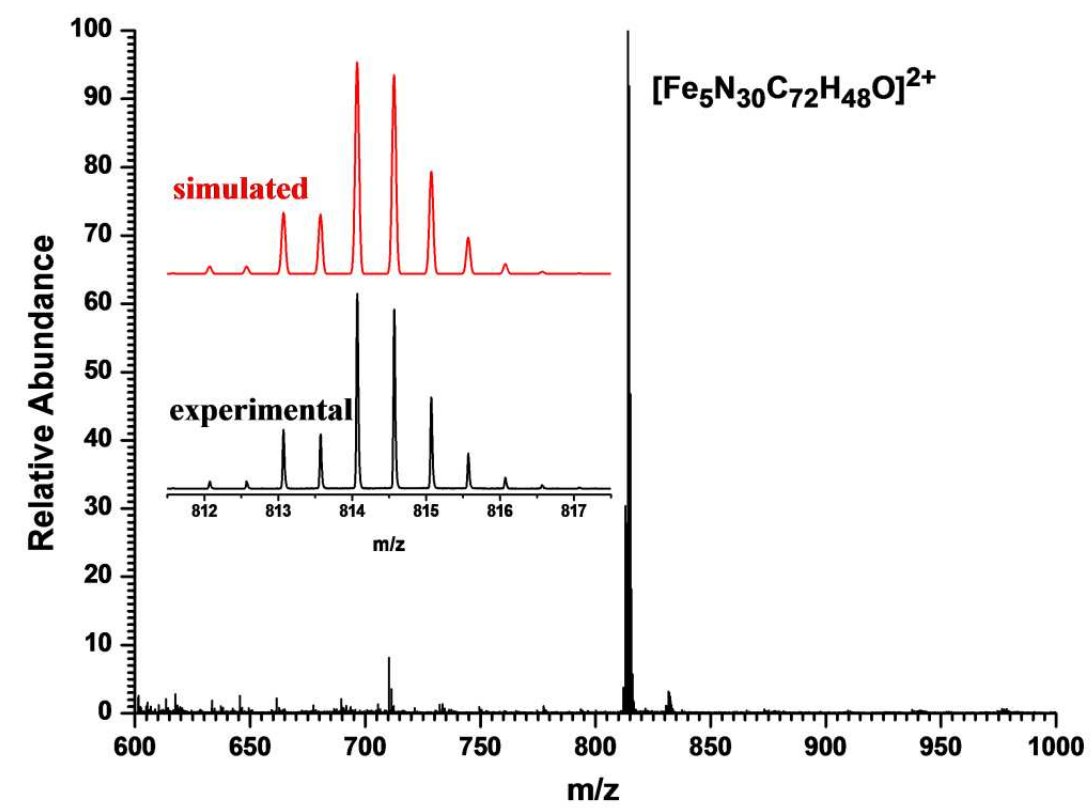

Figure S1. MS spectra of the $\left[\left\{\mathrm{Fe}^{\mathrm{II}}(\mu \text {-bpt })_{3}\right\}_{2} \mathrm{Fe}_{3}^{\mathrm{II}}\left(\mu_{3}-\mathrm{O}\right)\right]^{2+}$ cation in $\mathbf{1}$, the insets picture shows the experimental and simulated isotopic distributions.

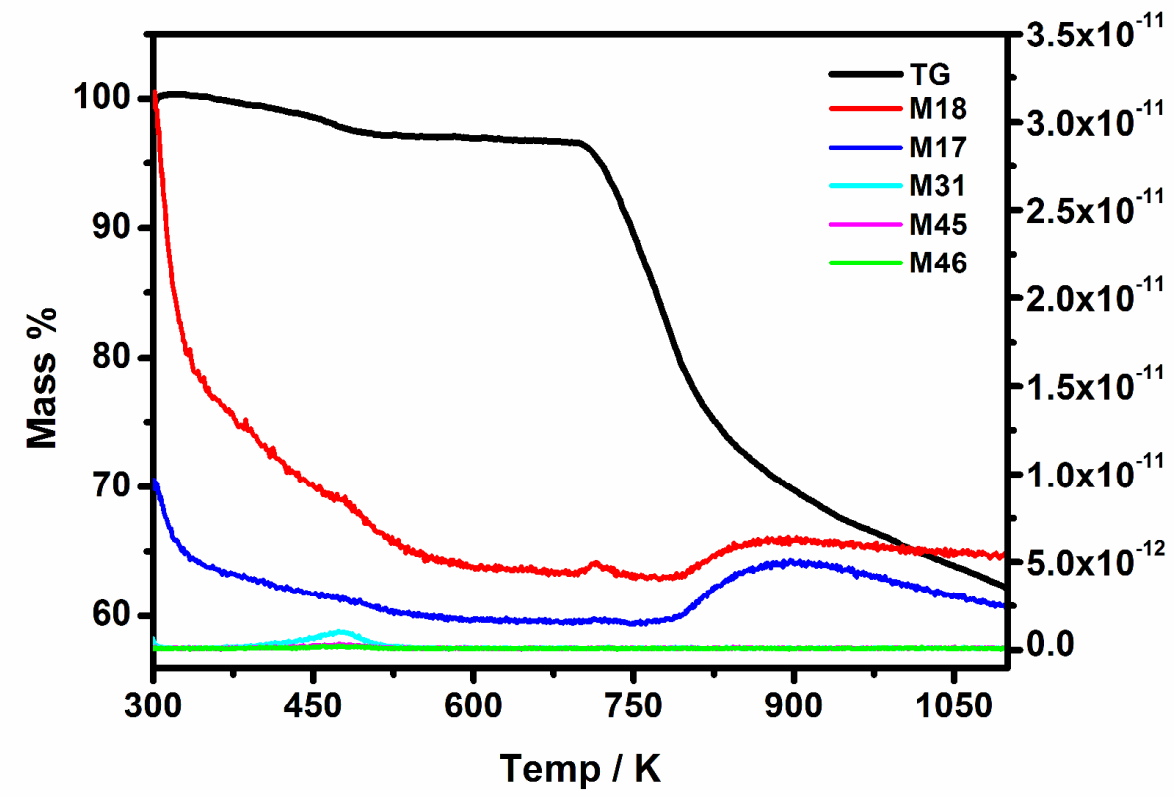

Figure S2. Thermogravimetric-mass spectroscopy analysis of $\mathbf{1}$ under $\mathrm{N}_{2}$ atmosphere $(10 \mathrm{~K}$ $\mathrm{min}^{-1}$ ). The fragment ions of mass spectrum for different solvents are referred to NIST Chemistry WebBook. $\mathrm{H}_{2} \mathrm{O}$ : M17, M18; EtOH: M31, M45, M46. 


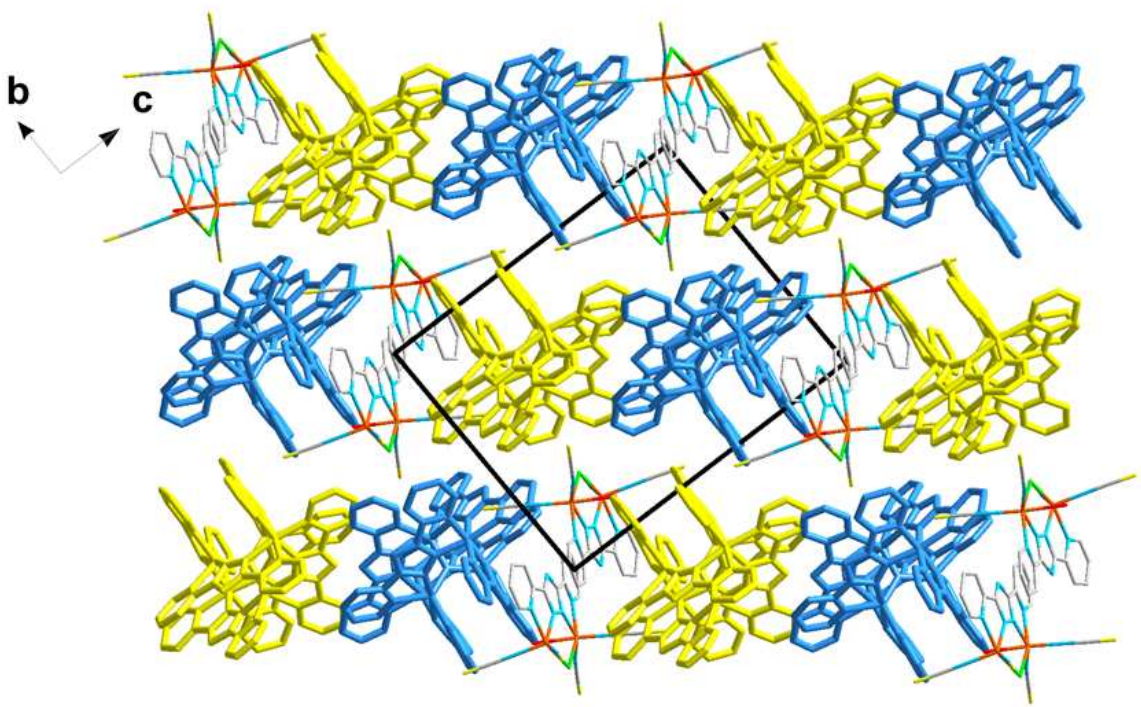

Figure S3. Packing diagram of $\mathbf{1}$ showing heterochiral layers. $\Delta, \Delta$-configuration (right-handed, $\mathrm{P}$ ) is shown in yellow strands, $\Lambda, \Lambda$-configuration (left-handed, M) in blue strands. 


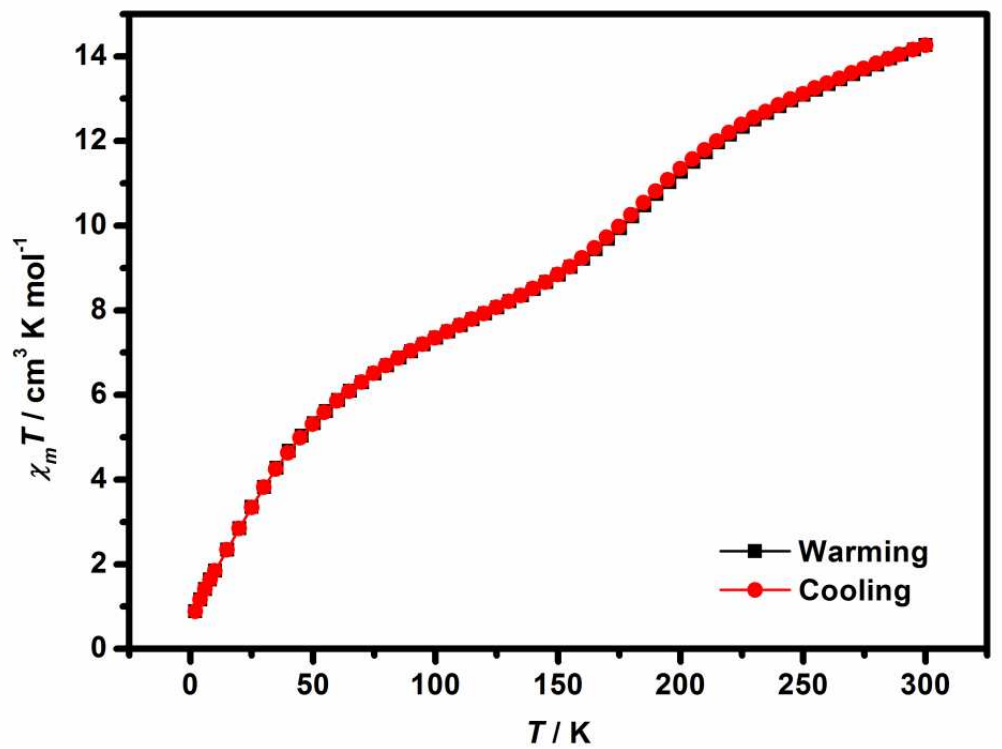

Figure S4. Plots of $\chi_{\mathrm{M}} T$ vs $T$ for $\mathbf{1}$. 
Table S1. The values of trigonality index $\tau$ for Fe3 Fe5 and Fe6.

\begin{tabular}{|l|l|l|l|l|}
\hline & Fe3 & Fe4 & Fe5 & Fe6 \\
\hline $293 \mathrm{~K}$ & 0.61 & 0.57 & 0.73 & 0.45 \\
\hline $150 \mathrm{~K}$ & 0.67 & 0.66 & 0.73 & 0.42 \\
\hline
\end{tabular}


Table S2. Intermolecular interactions around two apical [ $\left.\mathrm{Fe}^{\mathrm{II}}(\mathrm{bpt})_{3}\right]^{-}$units at $293 \mathrm{~K}(150 \mathrm{~K})$. The red and violet data are the interactions of pyridine and triazole parts coordinated to $\mathrm{Fe} 1$ and $\mathrm{Fe} 2$, respectively, while the black data are the interactions of pyridine parts coordinated to the $\left[\mathrm{Fe}_{3}^{\mathrm{II}}\left(\mu_{3}-\mathrm{O}\right)\right]^{4+}$ core.

\begin{tabular}{|c|c|c|c|c|c|c|c|c|}
\hline & & \multicolumn{3}{|c|}{ Hydrogen bonds } & \multicolumn{2}{|c|}{$\pi \cdots \pi$ interactions } & \multicolumn{2}{|c|}{ Short contacts } \\
\hline & & $\mathrm{D}-\mathrm{H} \cdots \mathrm{A}$ & $\mathrm{d}(\mathrm{D} \cdots \mathrm{A})$ & $<(\mathrm{DHA})$ & & & & \\
\hline \multirow{8}{*}{$\begin{array}{c}\mathrm{Fe} 1 \\
293 \mathrm{~K} \\
(150 \mathrm{~K})\end{array}$} & \multirow[t]{2}{*}{$\operatorname{bpt}^{-}(1)$} & $\mathrm{C} 2-\mathrm{H} 2 \cdots \mathrm{Cl1}^{\mathrm{a}}$ & $3.771(3.770)$ & $137.07(134.15)$ & & & C $3 \cdots N 37^{a}$ & $3.207(3.161)$ \\
\hline & & C9-H9 $\cdots$ S3 & $3.551(3.513)$ & $124.94(123.44)$ & & & $\mathrm{C} 10 \cdots \mathrm{C} 87$ & $3.226(3.186)$ \\
\hline & \multirow[t]{4}{*}{$\operatorname{bpt}^{-}(2)$} & O1W-H1WB ${ }^{\mathrm{b}} \cdots \mathrm{N} 10$ & $2.841(2.831)$ & $172.59(171.06)$ & $\mathrm{C} 15 \cdots \mathrm{C} 77^{\mathrm{b}}$ & $3.170(3.169)$ & & \\
\hline & & $\mathrm{C} 23-\mathrm{H} 23 \cdots \mathrm{S} 2^{\mathrm{c}}$ & $3.694(3.590)$ & $152.11(151.30)$ & $\mathrm{C} 16 \cdots \mathrm{C} 78^{\mathrm{b}}$ & $3.321(3.269)$ & & \\
\hline & & & & & $\mathrm{N} 10 \cdots \mathrm{C} 80^{\mathrm{b}}$ & $3.532(3.492)$ & & \\
\hline & & & & & $\mathrm{N} 10 \cdots \mathrm{C} 79^{\mathrm{b}}$ & $----(3.681)$ & & \\
\hline & \multirow[t]{2}{*}{$\operatorname{bpt}^{-}(3)$} & $\mathrm{C} 35-\mathrm{H} 35 \cdots \mathrm{S} 4^{\mathrm{d}}$ & $3.771(3.781)$ & $145.36(149.12)$ & $\mathrm{C} 34 \cdots \mathrm{C} 38^{\mathrm{d}}$ & $----(3.512)$ & $\mathrm{C} 27 \cdots \mathrm{S} 2$ & $3.395(3.293)$ \\
\hline & & $\mathrm{C} 37-\mathrm{H} 37 \cdots \mathrm{N} 25^{\mathrm{d}}$ & $----(3.313)$ & $----(122.49)$ & $\mathrm{N} 25 \cdots \mathrm{C} 52^{\mathrm{d}}$ & $----(3.782)$ & & \\
\hline \multirow{8}{*}{$\begin{array}{c}\mathrm{Fe} 2 \\
293 \mathrm{~K} \\
(150 \mathrm{~K})\end{array}$} & \multirow[t]{2}{*}{$\operatorname{bpt}^{-}(4)$} & $\mathrm{C} 40-\mathrm{H} 40 \cdots \mathrm{S} 3^{\mathrm{e}}$ & $3.630(3.672)$ & $153.55(157.20)$ & & & & \\
\hline & & $\mathrm{C} 47-\mathrm{H} 47 \cdots \mathrm{S} 2^{\mathrm{a}}$ & $3.887(3.833)$ & $175.82(177.28)$ & & & & \\
\hline & \multirow[t]{4}{*}{$\operatorname{bpt}^{-}(5)$} & $\mathrm{C} 49-\mathrm{H} 49 \cdots \mathrm{S} 1^{\mathrm{d}}$ & $3.737(3.674)$ & $150.62(144.40)$ & $\mathrm{C} 52 \cdots \mathrm{N} 25^{\mathrm{d}}$ & $3.772(3.782)$ & & \\
\hline & & $\mathrm{C} 50-\mathrm{H} 50 \cdots \mathrm{Cl1}^{\mathrm{d}}$ & $3.775(3.857)$ & $133.63(125.97)$ & & & & \\
\hline & & $\mathrm{C} 58-\mathrm{H} 58 \cdots \mathrm{O} 2$ & -----(3.336) & $----(134.11)$ & & & & \\
\hline & & 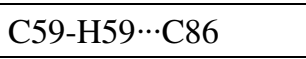 & $3.493(3.463)$ & $136.65(140.94)$ & & & & \\
\hline & \multirow[t]{2}{*}{$\operatorname{bpt}^{-}(6)$} & $\mathrm{C} 71-\mathrm{H} 71 \cdots \mathrm{S} 1^{\mathrm{b}}$ & $3.864(3.882)$ & $167.28(168.17)$ & $\mathrm{C} 66 \cdots \mathrm{C} 70^{\mathrm{f}}$ & 3.514(-----) & $\mathrm{C} 62 \cdots \mathrm{S} 1^{\mathrm{d}}$ & $3.347(3.324)$ \\
\hline & & $\mathrm{C} 75-\mathrm{H} 75 \cdots \mathrm{N} 20^{\mathrm{b}}$ & $----(3.422)$ & $-----(131.64)$ & C67 $\cdots$ C69 ${ }^{f}$ & $(----) 3.383$ & $\mathrm{C} 76 \cdots \mathrm{N} 38^{\mathrm{g}}$ & $-----(3.330)$ \\
\hline
\end{tabular}

symmetry codes: a) 1-x, 1-y, -z; b) x, 1+y, z; c) -1+x, 1+y, z; d) -x, 1-y, 1-z; e) -x, 1-y, -z; f) -x, 2-y, 1-z; g) 1-x, -y -z. 
Table S3. Mössbauer hyperfine parameters of 1, derived from the fitting procedure of the low-temperature and room-temperature Mössbauer spectra, where $\delta$ is the isomer shift, $\Delta E_{\mathrm{Q}}$ is the quadrupole splitting, and RA is the relative spectral area of individual spectral components.

\begin{tabular}{|c|c|c|c|c|c|}
\hline $\begin{array}{c}\text { Temperature } \\
\text { (K) }\end{array}$ & Component & $\begin{array}{c}\delta \\
\pm 0.01 \\
(\mathrm{~mm} / \mathrm{s})\end{array}$ & $\begin{array}{c}\Delta E_{Q} \\
\pm 0.01 \\
(\mathrm{~mm} / \mathrm{s})\end{array}$ & $\begin{array}{l}\text { RA } \\
\pm 1 \\
(\%)\end{array}$ & Assignment \\
\hline \multirow[t]{4}{*}{20} & Doublet & 1.02 & 2.35 & 0.43 & $\mathrm{HS}$, central $\left[\mathrm{Fe}_{3}\left(\mu_{3}-\mathrm{O}\right)\right]^{4+}$ \\
\hline & Doublet & 0.97 & 2.63 & 0.14 & \multirow{2}{*}{$\mathrm{HS},\left[\mathrm{Fe}_{2} \mathrm{Cl}(\mathrm{bpt})\left(\mathrm{H}_{2} \mathrm{O}\right)(\mathrm{NCS})_{4}\right]^{2-}$} \\
\hline & Doublet & 1.11 & 2.34 & 0.14 & \\
\hline & Doublet & 0.48 & 0.24 & 0.29 & $\mathrm{LS}, \mathrm{Fe} 1$ and $\mathrm{Fe} 2$ \\
\hline \multirow[t]{5}{*}{290} & Doublet & 0.90 & 2.31 & 0.43 & HS, central $\left[\mathrm{Fe}_{3}\left(\mu_{3}-\mathrm{O}\right)\right]^{4+}$ \\
\hline & Doublet & 1.22 & 1.84 & 0.14 & \multirow{2}{*}{$\mathrm{HS},\left[\mathrm{Fe}_{2} \mathrm{Cl}(\mathrm{bpt})\left(\mathrm{H}_{2} \mathrm{O}\right)(\mathrm{NCS})_{4}\right]^{2-}$} \\
\hline & Doublet & 0.79 & 2.40 & 0.14 & \\
\hline & Doublet & 0.99 & 0.84 & 0.14 & $\mathrm{HS}, \mathrm{Fe} 2(\mathrm{SCO})$ \\
\hline & Doublet & 0.40 & 0.20 & 0.14 & $\mathrm{LS} 1, \mathrm{Fe} 1$ \\
\hline
\end{tabular}

